\title{
Negative Mobility of Single Domain Wall in Magnetic Microwires
}

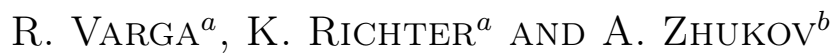 \\ ${ }^{a}$ Institute of Physics, Faculty of Sciences, P.J. Safarik University \\ Park Angelinum 9, 04154 Kosice, Slovakia \\ ${ }^{b}$ Dept. Fisica de Materiales, Fac. Quimica, UPV/EHU, San Sebastian, Spain
}

\begin{abstract}
Here, we present the domain wall dynamics in thin magnetic wires that exhibit even negative mobility regime. Such a regime is well below the Walker limit and is a result of structural relaxation. It disappears at high frequencies and it can be enhanced by application of mechanical stress. Moreover, the domain wall velocity was found to be almost field-independent at certain measuring conditions. Anyway, the domain wall velocity remains quite high $(>450 \mathrm{~m} / \mathrm{s})$ in this regime.
\end{abstract}

PACS numbers: 75.60.Ej, 75.60.Jk

\section{Introduction}

The domain wall dynamics in glass-coated microwires has revealed many new interesting phenomena [1]. Either it is a new contribution to the domain wall damping, fast domain wall velocity [2] that can overcome the sound speed [3], or negative critical propagation field [1].

Structural relaxation can effectively influence the domain wall dynamics. The domain wall damping can be increased or decreased by more than one order by simply changing the frequency of the measurements [4].

Here we present another interesting phenomenon in the domain wall dynamics in magnetic microwires. Having the domain structure stabilized, the domain wall dynamics is characterized by the negative domain wall mobility at low fields. Such effect can be tuned either by the frequency of exciting magnetic field, or by the stress applied on the microwire during the measurement.

\section{Experimental}

Amorphous glass-coated $\mathrm{Fe}_{49.6} \mathrm{Ni}_{27.9} \mathrm{Si}_{7.5} \mathrm{~B}_{15} \mathrm{mi}-$ crowire was produced by the Taylor-Ulitovski method. The diameter of metal core is $15 \mu \mathrm{m}$ and total diameter is $32 \mu \mathrm{m}$. The length of microwire was $10 \mathrm{~cm}$. The Curie temperature of the microwire is $697 \mathrm{~K}$.

The microwire was annealed at $573 \mathrm{~K}$ in order to release the stress introduced during production and to stabilize its domain structure. The domain wall velocity measurements were performed by Sixtus-Tonks method at the temperature $125 \mathrm{~K}$, where the effect of structural relaxation damping is the strongest [5].

\section{Results and discussion}

The domain wall velocity, measured at various frequencies, is shown in Fig. 1. At high frequencies, the domain wall velocity $v$ is proportional to the applied magnetic field $H$ according to [1]:

$$
v=S\left(H-H_{0}\right),
$$

where $S$ is the domain wall mobility constant (defined as the slope of $v \sim f(H)$ dependence: $S=v / H)$ and $H_{0}$ is so-called critical propagation field. Decreasing the frequency of the applied magnetic field down to $500 \mathrm{~Hz}$, minimum of the domain wall velocity appears at the field of $350 \mathrm{~A} / \mathrm{m}$. Below this field, the domain wall mobility $S$ is negative. The lower is the frequency of the measuring field, the higher is the field at which such minimum appears. The negative domain wall mobility is a curious phenomenon: the higher is the force acting on the domain wall (through the magnetic field), the lower is the domain wall velocity. Negative domain wall velocity has already been observed above the so-called Walker limit [5], when the domain wall structure changes during the propagation and this effect costs energy. However, it appears at much higher field than in our case. Hence, the negative mobility must have another origin.

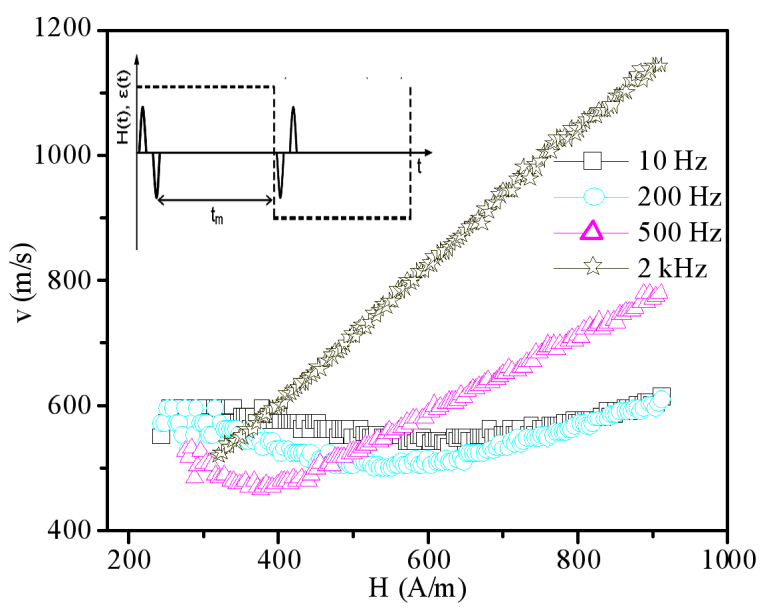

Fig. 1. Domain wall velocity $v$ as a function of the magnetic field amplitude $H$. Frequency of applied field as a parameter. 
One possible explanation can be offered in terms of complex domain structure of amorphous glass coated microwires and its stabilization by heat treatment. It consists of single core domain with axially oriented magnetization that is surrounded by the radial domain structure (Fig. 2). Actual diameter of the inner core domain is given by the stress distribution and applied magnetic field.

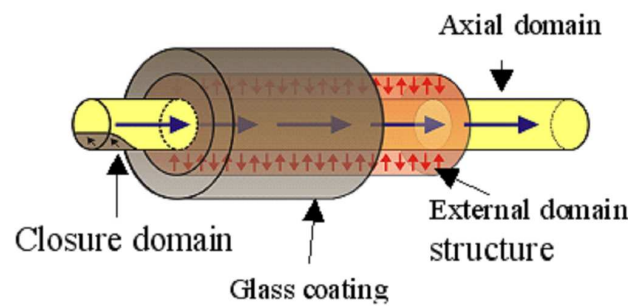

Fig. 2. Domain structure of amorphous microwire.

Such a domain structure is stabilized by an annealing at $573 \mathrm{~K}$. However, when the axial field is applied during the domain wall velocity measurement, the radius of axial domain increases. If there is a time (at low frequencies), new domain structure (with higher diameter of the core domain) relaxes and the surface of the domain wall propagating along microwires increases. This costs energy and, hence, the domain wall propagation is slower. Such effect is observed up to certain field (where the minimum of the velocity appears). Above this field, the diameter of the core domain is saturated and increase of the field results in the increase of the domain wall velocity according to Eq. (1).

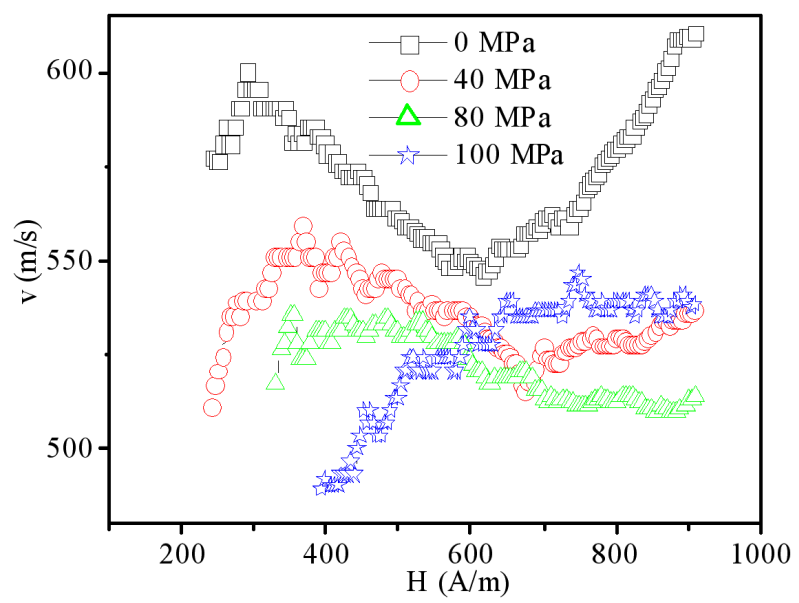

Fig. 3. Domain wall velocity $v$ as a function of the magnetic field amplitude $H$ measured at $10 \mathrm{~Hz}$. Axial stress as a parameter.

The effect is sensible to the frequency of the applied field. One should take into account that the domain wall propagates immediately after the direction of the field is reversed. After the domain wall propagation, the domain structure of the microwires is exposed to the field and has a time $t_{\mathrm{m}}$ to relax (see the inset of Fig. 1). At high frequencies, the time is short and the domain structure has no time to stabilize itself. The lower is the frequency of applied field, the longer is the time for relaxation and stabilization of thicker core domain and the effect of negative mobility is enhanced.

Such model is confirmed also by the measurement of the domain wall velocity under the stress applied. The negative mobility $S$ can be controlled by the applied axial stress. The higher is the stress applied, the higher is the diameter. Hence the region with negative mobility is enhanced and shifted to the higher fields (Fig. 3). Moreover, at certain condition, the range with zero domain wall mobility is observed (in the field range 600-900 A/m), when the applied stress amplitude is $100 \mathrm{MPa}$. Although the domain wall mobility is zero, the domain wall velocity is quite high $(540 \mathrm{~m} / \mathrm{s})$. This gives us possibility to stabilize the domain wall propagation in a wide range of the field.

\section{Conclusions}

We have studied the domain wall dynamics in the amorphous glass coated microwires with the domain structure stabilized by the heat treatment. The region with negative domain wall mobility has been observed. Such effect can be tuned by the frequency of applied field as well as by the stress applied in axial direction. Under selected condition, the region with zero domain wall mobility can be obtained. This gives us possibility to stabilize the domain wall propagation at quite high velocities (above $500 \mathrm{~m} / \mathrm{s}$ ).

\section{Acknowledgments}

This work was supported by the project NanoCEXmat No. ITMS 26220120019, Slovak VEGA grant No. 1/0076/09 and project MVTS 6RP/Manunet/UPJS/08.

\section{References}

[1] R. Varga, K.L. Garcia, M. Vázquez, P. Vojtanik, Phys. Rev. Lett. 94, 017201 (2005).

[2] J.D. Santos, Á. Ruiz, R.F. Cobos, I. Ribot, V. Vega, P. Álvarez, M.L. Sánchez, J.L. Sánchez, Ll., V.M. de la Prida, B. Hernando, Phys. Status Solidi A 206. 618 (2009).

[3] K. Richter, R. Varga, G. Infante, G.A. Badini-Confalonieri, M. Vazquez, IEEE Trans. Magn. 46, 210 (2010).

[4] G. Infante, R. Varga, G.A. Badini-Confalonieri, M. Vázquez, Appl. Phys. Lett. 95, 012503 (2009).

[5] N.L. Schryer, L.R. Walker, J. Appl. Phys. 45, 5406 (1974). 